\title{
Implantando o Modelo CMMI em uma Empresa de Software de Pequeno Porte Jovem e Imatura
}

\author{
Heron Vieira Aguiar', Ana Cristina Rouiller ${ }^{2}$, Renata Teles Moreira ${ }^{1}$, Alexandre \\ Marcos Lins de Vasconcelos ${ }^{3}$, Sandro Ronaldo Bezerra Oliveira ${ }^{4}$ \\ ${ }^{1}$ SWQuality Consultoria e Sistemas LTDA \\ Campus Histórico da UFLA, CEP 37200-000 Lavras/MG - Brasil \\ ${ }^{2}$ Departamento de Ciência da Computação-DCC, \\ Universidade Federal de Lavras - UFLA \\ Cxp Postal 37 - CEP 37200-000 Lavras/MG - Brasil \\ ${ }^{3}$ Centro de Informática - Cin, Universidade Federal de Pernambuco - UFPE \\ Cxp Postal 7851 - CEP 50732-970 Recife/PE - Brasil \\ ${ }^{4}$ Centro de Ciências Exatas e Tecnologia, Universidade da Amazônia - UNAMA \\ Av. Alcindo Cacela, 287 - CEP 66060-000 Belém/PA - Brasil \\ heron@swquality.com.br, ana@ufla.br, renata@swquality.com.br, \\ amlv@cin.ufpe.br, sandro@cci.unama.br
}

\begin{abstract}
This paper presents a case about software process improvement with CMMI in a small company. This company has some adverse characteristic such as: (1) It was created 2 years ago and (2) Your staff is composed by students of Computer Science. This work intends to check if one model make for big companies (like CMMI model) can be used successfully by the small companies.
\end{abstract}

Resumo. Neste artigo é apresentado um relato de experiência sobre a implantação de um programa de melhoria com CMMI em uma empresa de software de pequeno porte. Esta empresa além de possuir as dificuldades inerentes de seu porte ainda tem algumas características adversas como: ser jovem (menos de 2 anos de existência) e imatura (composta em sua maioria por alunos que ainda cursam Ciência da Computação). A intenção deste trabalho é averiguar o quão é aplicável, para uma empresa de pequeno porte, o uso de um modelo de qualidade, originalmente construído para empresas departamentalizadas e de grande porte.

\section{Introdução}

Neste início de século XXI, fatores como a globalização da economia e a maior competitividade do mercado têm gerado inúmeros desafios para as empresas. No caso das empresas relacionadas com desenvolvimento de software, construir tais sistemas em tempo hábil, com custos razoáveis e qualidade adequada tornou-se fundamental [CÂNDIDO 2004].

Para empresas de software obterem sucesso, é preciso existir harmonia entre seus processos de software, focando: pessoas, produtos, processos e projetos [HUMPHREY 1989]. Sob a ótica de processo, segundo Pressman [PRESSMAN 2002], a falta de adoção de métodos, ferramentas e procedimentos no desenvolvimento de software, tem alcançado números expressivos de projetos não concluídos, e projetos concluídos que não atendem às necessidades do cliente. Segundo uma pesquisa 
realizada pelo Ministério da Ciência e Tecnologia (MCT) [MCT 2001], as empresas de software têm utilizado pouco as normas e modelos de qualidade de processo no Brasil. Analisando os dados desta pesquisa, pode-se constatar a falta e/ou pouca cultura e maturidade da indústria de software brasileira em qualidade de processo.

Dentre as normas e modelos de qualidade de processo de software, o Capability Maturity Model Integration (CMMI)[CMMI 2005] merece destaque, pois o mesmo é consagrado mundialmente. De acordo com uma pesquisa do MCT, citada pela Sociedade para Promoção da Excelência do Software Brasileiro (Softex)[SOFTEX 2004], em 2003 foram "certificadas" apenas 30 empresas brasileiras no CMM. Este número, se comparado ao de países concorrentes neste segmento, deixa o Brasil em desvantagem.

Nas empresas de software de pequeno porte ${ }^{1}$, que em 2001 representavam $58 \%$ das empresas de software brasileiras [MCT 2001], além das dificuldades comuns de implementação, segundo Rouiller [ROUILLER 2001] outros fatores ainda agravam os problemas de uso de processos em empresas de software de pequeno porte, tais como: recursos pessoais e financeiros limitados, falta e/ou pouca cultura em processos, pouco treinamento em engenharia de software, imaturidade metodológica, crescimento ocorrido por demanda, falta de experiência administrativa por parte dos gerentes e diretores e falta de definição das metas organizacionais. São fatores como esses que impedem o crescimento do número de empresas de pequeno porte utilizando processos.

\subsection{Objetivo do Trabalho}

O principal objetivo deste trabalho é contribuir com estado da arte em Qualidade de Software, no que se refere à pesquisa de implantação de modelos como o CMMI em empresas de pequeno porte, considerando ainda pontos extremos como a imaturidade da organização e seu tempo de existência. A sua realização se justifica por não existir um número significativo de trabalhos que comprovem a aplicabilidade do CMMI em empresas de pequeno porte, embora não exista nenhuma restrição descrita no modelo.

\subsection{Caracterização da Empresa do Estudo}

A empresa, que foi utilizada como estudo de caso atua há menos de dois anos no mercado. Possui 27 funcionários, sendo quatro recém formados, vinte e um estagiários e mais duas secretárias. A área de desenvolvimento é composta por recém-formados e alunos de graduação, ambos com pouca experiência. Essas características fazem dela uma empresa jovem e imatura na área de desenvolvimento de software.

O ambiente, cujo estudo foi aplicado, compreende as seguintes áreas de negócio da organização:

Fábrica de Software: desenvolve projetos e produtos sob encomenda para clientes (inclusive para outras empresas de software);

$\checkmark$ Ensino a Distância: editora e elabora conteúdo para cursos a distância. Administra e customiza ambiente virtual de ensino;

\section{Modelo CMMI}

A sigla CMMI [CMMI 2005] representa as iniciais de Capability Maturity Model Integration e nomeia tanto um projeto, quanto os modelos resultantes deste projeto. $\mathrm{O}$ Projeto CMMI, que pode ser traduzido como Projeto de Integração dos Modelos de Maturidade da Capacidade, está sendo executado pelo Software Engineering Institute (SEI), em cooperação com a indústria e governo, para consolidar um framework para

\footnotetext{
${ }^{1}$ Segundo o MCT é considerado micro as empresas com até 10 funcionários, e pequena as com até 50 funcionários. Estas empresas são referenciadas no trabalho como empresas de pequeno porte.
} 
modelos, evoluir e integrar modelos desenvolvidos pelo SEI (inicialmente os modelos SW-CMM, SE-CMM e IPD-CMM), e gerar seus produtos associados, incluindo material de treinamento e método de avaliação.

\subsection{Arquitetura do Modelo}

A arquitetura do CMMI é composta, basicamente, pela definição de um conjunto de áreas de processo, organizadas em duas representações diferentes: uma como um modelo por estágio, semelhante ao SW-CMM, e outra como um modelo contínuo (semelhante à ISO/IEC 15504[ISO 2004]). A versão atual é composta por 25 áreas de processo.

Cada área de processo é definida no modelo por meio da descrição de seu propósito, notas introdutórias, relacionamentos com outras áreas, metas específicas, metas genéricas, práticas e subpráticas específicas, práticas e subpráticas genéricas, produtos de trabalho típicos e referências para outros elementos do modelo relacionados.

$\mathrm{Na}$ representação por estágio, as 25 áreas de processo estão agrupadas em 4 níveis de maturidade: níveis $2,3,4$ e 5 . O nível 1 não contém nenhuma área de processo, e a única exigência para que a empresa de software seja qualificada neste nível é a sua própria existência. Na representação contínua, as mesmas 25 áreas de processo estão agrupadas em quatro grupos (gerência de processos, gerência de projetos, engenharia e suporte) e são definidos seis níveis de capacidade de processo.

\section{Metodologia para Criação e Implementação do Processo}

Para definir a metodologia de criação e implementação do processo, foi realizada uma pesquisa pela empresa sobre a melhor forma de criar, manter e institucionalizar um processo. O resultado desta pesquisa foi a escolha do modelo IDEAL ${ }^{\text {SM }}$ [IDEAL 2004], como base para a criação da metodologia. São apresentadas a seguir as etapas que compõem a metodologia e como ela foi aplicada na organização.

\subsection{Etapa de Iniciação}

Nesta etapa foram realizadas reuniões com a alta direção para a aprovação do trabalho na empresa e a definição dos recursos necessários para o desenvolvimento.

Em seguida, foram realizadas pesquisas para escolher o modelo que seria aplicado ao processo. Baseado nas características do CMMI, optou-se pela utilização do modelo CMMI-SE/SW como base para a criação do processo. Outro fator que influenciou a escolha foi o reconhecimento que o CMMI possui internacionalmente. A representação escolhida foi a estagiada, por descrever a sequiência de execução das áreas de processo agrupando-as em níveis. Alcançando cada nível garante-se uma base adequada de melhorias para o próximo nível.

O próximo passo para a criação do processo foi a escolha dos possíveis funcionários da empresa que estariam aptos a participar do Software Engineering Process Group(SEPG). Após a seleção, o SEPG ficou estruturado da seguinte maneira:

- Gerente do Projeto: alocado para o projeto em tempo integral. Responsável pela gerência do projeto; planejar, coordenar e participar das atividades de definição, validação e institucionalização do processo;

- Líder do Projeto: alocado para o projeto em tempo parcial. Responsável por auxiliar o Gerente do Projeto. Foi atribuída também a gerência dos treinamentos necessários na empresa;

- Membro Temporário: alocado para o projeto esporadicamente. Responsável por auxiliar na definição de determinada fase do processo quando solicitado. O critério 
básico para escolha deste funcionário é a relação entre a sua área de atuação na empresa e a fase.

\subsection{Etapa de Diagnóstico}

Para elaborar o planejamento das atividades e saber o estado atual da empresa, com relação aos elementos requeridos no nível 2 do modelo CMMI, foi feito um diagnóstico da empresa. Este diagnóstico foi realizado com a participação do Gerente e do Líder do Projeto, pois estes possuíam visões técnicas, gerenciais e administrativas dos projetos executados pela empresa.

O diagnóstico foi feito através de uma lista de verificação que continha todas as práticas descritas na versão estagiado do modelo CMMI nível 2. Os critérios para pontuação basearam-se no método de avaliação da norma ISO/IEC 15504. A aderência da empresa as áreas de processos pode ser observada na Tabela 1. De acordo com os resultados obtidos, a empresa encontrava-se no nível 1(Inicial) de maturidade do CMMI. A PA SAM não foi avaliada por não se aplicar ao negócio da empresa e também por não ser obrigatória em uma avaliação oficial do nível 2 do CMMI.

Tabela 1: Aderência da empresa ao CMMI nível 2

\begin{tabular}{|c|c|c|c|c|c|c|}
\hline PA & REQM & PP & PMC & MA & PPQA & CM \\
\hline Nota & $67 \%$ & $34 \%$ & $18 \%$ & $0 \%$ & $23 \%$ & $27 \%$ \\
\hline
\end{tabular}

\subsection{Etapa de Estabelecimento}

Para uma melhor condução do projeto fez-se necessária a elaboração de um Plano de Projeto, onde foram descritas as atividades para a execução do projeto, a estimativa de duração e um cronograma. O Plano de Projeto serviu também como base para o acompanhamento do projeto durante a sua execução.

Para facilitar a institucionalização e o uso do processo na empresa, chegou-se no consenso de que deveria ser criada uma versão navegável do processo. Para atender a este requisito, foi escolhida a ferramenta Microsoft Visio, por possuir uma funcionalidade que salva o desenho do processo no formato html (página para internet, sites). Através da versão navegável é possível visualizar todas as fases, etapas, atividades do processo e documentos de referência, bastando estar conectado à internet. É possível também ter acesso a todos os templates utilizados pelo processo.

Para definir as atividades que iriam compor o Plano de Projeto tomou-se como exemplo o Modelo de Ciclo de Vida de Projetos. Segundo Project Management Body of Knowledge (PMBOK)[PMBOK 2000], as organizações que desenvolvem projetos usualmente dividem-nos em várias fases visando um melhor controle gerencial e uma ligação mais adequada de cada projeto às atividades de caráter repetitivo e contínuo. A este conjunto de fases do projeto é dado o nome de Modelo de Ciclo de Vida de Projetos. Cada fase do projeto é marcada pela conclusão de um ou mais produto de trabalho (work product), tangível e verificável. Desta forma, chegou-se ao consenso de que a criação do processo orientado ao Modelo de Ciclo de Vida de Projetos seria mais vantajosa para o entendimento do processo.

O Modelo de Ciclo de Vida de Projetos definido para a empresa, apresentado na Figura 3.1 abaixo, ficou divido em quatro fases: Prospecção, Planejamento, Desenvolvimento e Fechamento. Estas fases foram definidas baseando-se no ciclo de vida dos projetos realizados na empresa e serão detalhadas na próxima etapa. Para apoiar o processo definido dentro das fases do Modelo de Ciclo de Vida de Projetos foram acrescentadas as Áreas de Processo de Suporte Básico: Measurement and Analysis(MA), Configuration Management (CM) e Process and Product Quality Assurance(PPQA). 
Após a criação do processo, deveria ser realizada uma avaliação em que fosse indicado o grau de aderência do processo ao CMMI. Para validar e institucionalizar o processo, deveria executado um treinamento no processo para os stakeholders ${ }^{2}$ envolvidos. Em seguida, seriam escolhidos dois projetos, nomeados como projetos pilotos, onde seriam verificados o uso do processo, as inconsistências e erros existentes. Neste momento, seriam acrescidas as práticas que não foram atendidas na avaliação. Optou-se por institucionalizar o processo somente quando todas as fases estivessem definidas e validadas.

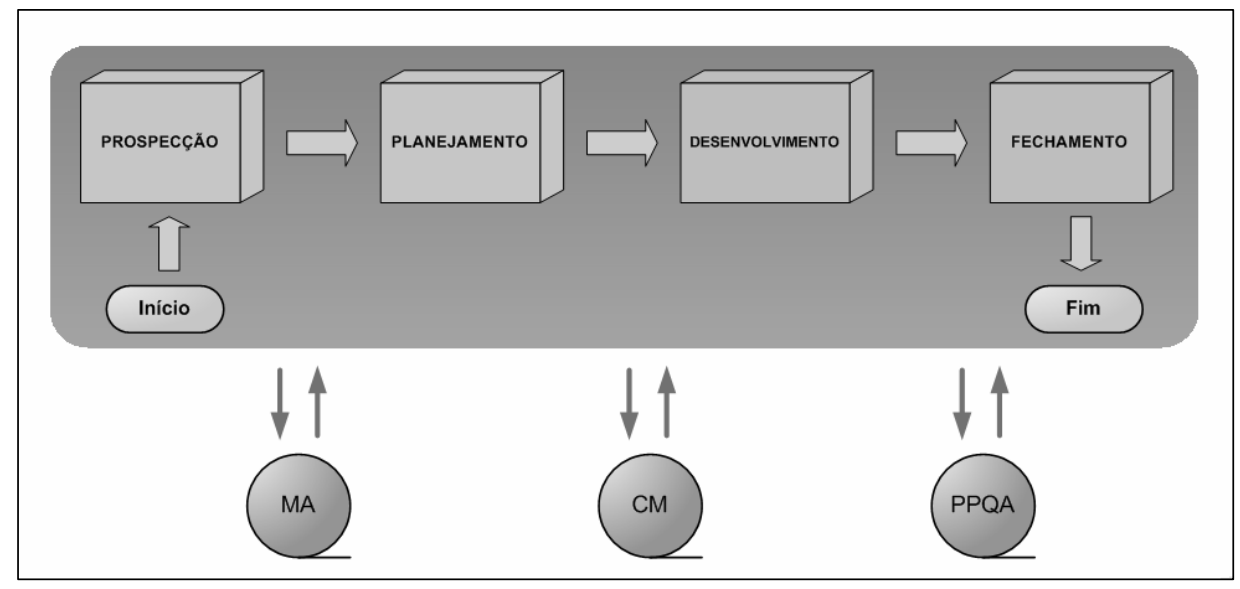

Figura 3.1: Modelo de Ciclo de Vida de Projetos

\subsection{Etapa de Ação}

A criação do processo foi dividida com base nas fases do Modelo de Ciclo de Vida de Projetos. Para cada fase foi eleito um workteam ${ }^{4}$. Este workteam era composto pelo Gerente e o Líder do projeto de Implantação, que eram os responsáveis por conduzir as reuniões, e os Membros Temporários escolhidos para a definição da fase. Nas reuniões de criação do processo eram mapeados procedimentos existentes na empresa, acrescentando as práticas do CMMI.

Para cada fase foram definidas quais as atividades eram necessárias para que os produtos de trabalho fossem gerados. Para cada atividade, era atribuído um, ou mais, responsável pela execução da mesma. Normalmente para cada atividade são atribuídos os artefatos de entrada e de saída, podendo existir atividades que não possuam artefatos. Os artefatos de entrada podem ser templates de documentos, documentos preenchidos, padrões do processo, elementos informativos. Os artefatos de saída podem ser um, ou mais, artefatos de entrada atualizados ou preenchidos. detalhes.

A seguir são apresentadas as fases do Modelo de Ciclo de Vida de Projeto em

\subsubsection{Fases do Modelo de Ciclo de Vida de Projeto}

A Fase de Prospecção é caracterizada pelo atendimento de uma solicitação de projeto feita pelo cliente. Após a definição do escopo do projeto, é elaborada uma proposta para o cliente. Nesta proposta, estará delimitado o escopo, o cronograma de execução do projeto, o valor do projeto e as restrições contratuais. A aprovação desta proposta pelo

\footnotetext{
${ }^{2}$ Grupo ou indivíduo afetado de alguma maneira pelo empreendimento. Inclui entre outros: membros do projeto, fornecedores, clientes, usuários finais.

${ }^{4}$ Equipe de trabalho formada pelos funcionários selecionados para participar da definição do processo.
} 
cliente implica na mudança para a Fase de Planejamento. A Fase de Prospecção está dividida em 4 etapas (Vide Figura 3.2): Atendimento, Análise, Proposta e Negociação.

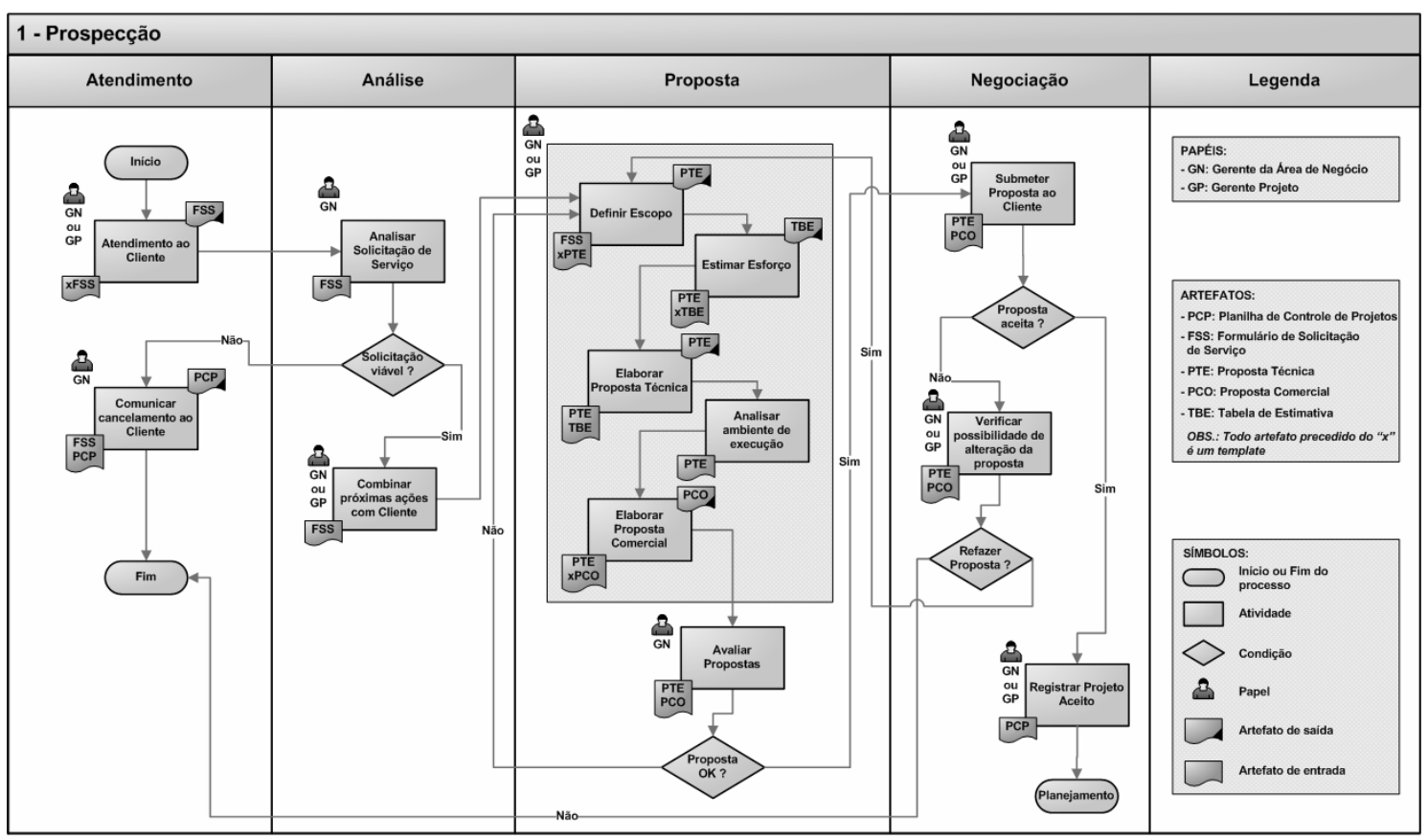

Figura 3.2: Fase de Prospecção

$\mathrm{Na}$ etapa de Atendimento é realizado o atendimento ao cliente e registrada a solicitação do projeto. Na etapa de Análise é realizada uma análise de viabilidade do projeto para a empresa. São considerados aspectos como, por exemplo: tecnologia necessária para execução do projeto, oportunidade de mercado, tipo de cliente, quantidade de projetos em andamento. Na etapa de Proposta, após aprovação da viabilidade, é elaborada uma proposta técnica e comercial contendo: escopo do projeto, estimativa de esforço e custo, cronograma de execução do projeto e as restrições contratuais. Na etapa de Negociação, a proposta é submetida ao cliente. Se a proposta for aprovada o projeto passa para Fase de Planejamento. Caso a proposta não seja aceita, dependendo do motivo relato pelo cliente, é analisado a possibilidade de se refazer a proposta.

Para complementar as informações do fluxo do processo foi criado um documento de referência para as atividades das fases. Neste documento, para cada atividade são apresentados:

- Uma descrição sobre sua finalidade;

- Quais são os artefatos de entrada e saída;

- Quem é o responsável por executá-la;

- Quais são as ferramentas que devem ser utilizadas;

- E um passo-a-passo de como executar a atividade;

Na Fase de Planejamento o escopo do projeto acordado na proposta é refinado e em seguida é feito um novo planejamento. Nesta fase, normalmente, o escopo do projeto não está muito detalhado, o que pode gerar desgastes ou até prejuízos entre as partes envolvidas no momento de homologação do projeto. Para minimizar os riscos, deve-se detalhar o escopo num nível atômico, abrangendo uma quantidade de detalhes que dê segurança para empresa, e após o detalhamento obter o aval do cliente com esta nova versão do escopo.

Outro fator preponderante para a entrega do projeto de acordo com o estabelecido na proposta é a elaboração de um plano de projeto. Este plano contém as diretrizes que todo projeto dentro da empresa deve seguir, além dos específicos do 
projeto. Os passos para construção deste plano são: estimar o esforço, o custo, identificar os riscos e os recursos da empresa e do cliente que serão necessários para execução do projeto.

Para finalizar o plano devem ser definidas as iterações do projeto. Após a aprovação do plano pela alta gerência deve-se obter o compromisso do cliente com o plano. Esta ação faz com que o cliente esteja ciente do que foi planejado, assumindo as responsabilidades a ele atribuídas e também o aproximando do desenvolvimento e dos possíveis problemas que possam acontecer.

A Fase de Desenvolvimento de um projeto é o momento em que as atividades definidas na Fase de Planejamento são executadas.O primeiro passo desta fase é definir a arquitetura do projeto. Em seguida são elaborados o projeto conceitual, o lógico e o físico. Após estas atividades, são produzidos os códigos fontes e os casos de teste. Para a conclusão da fase, o código é testado, e em seguida homologado no ambiente definido pelo cliente, podendo também ser homologado no ambiente de desenvolvimento da empresa.

Para conclusão do projeto, na Fase de Fechamento, após a homologação do projeto na Fase de Desenvolvimento, o projeto é implantado em um ambiente de teste no cliente posteriormente, no ambiente de produção. Também são planejadas as atividades de garantia e manutenção, caso existam.

\subsection{Etapa de Aprendizagem}

Embora o processo ainda não tenha sido totalmente institucionalizado, diversas atividades e procedimentos já estão sendo utilizados na empresa. Alguns procedimentos incorporados a projetos em execução se mostraram eficientes, e com boa aceitação pelos stakeholders.

Após a definição do processo abrangendo todas as Fases do Modelo de Ciclo de Vida de Projetos foi realizada uma auto-avaliação pelo SEPG. Nesta avaliação o processo foi classificado com parcialmente aderente ao CMMI.

Acredita-se que a institucionalização ocorrerá sem grandes impactos na empresa, pois o processo manteve características originais da organização. Espera-se que após o término dos projetos pilotos o processo esteja muito próximo do cumprimento de todas as exigências do nível 2 do CMMI.

\section{Conclusões e Trabalhos Futuros}

Neste trabalho foi analisada a implantação de um processo de software em empresas de pequeno porte utilizando o modelo CMMI. Foi considerado para este estudo um caso extremo: imaturidade da organização e seu pouco tempo de existência.

Embora a pesquisa ainda esteja em fase preliminar, neste artigo já pode ser apresentado alguns resultados da primeira fase de implantação do CMMI que teve duração de 6 meses. Partes dos processos já estão sendo institucionalizados e a próxima fase será a implantação do processo como um todo nos projetos pilotos, que ocorrerá no primeiro semestre 2005. Nesta ocasião serão realizados os ajustes para o que o mesmo se aproxime da aderência integral ao nível 2 do CMMI.

Com o resultado da avaliação da primeira versão e uso do processo criado, no qual foi classificado como parcialmente aderente, observou-se que é possível uma empresa de pequeno porte utilizar o CMMI. Um fator positivo, determinante para o sucesso do projeto, foi o interesse da maioria dos membros da empresa na adoção do CMMI. Como a maioria dos membros participou ativamente, não houve resistência na adoção dos processos.

Notou-se que, apesar da maioria das pesquisas na área apontarem para grandes dificuldade do uso do modelo CMMI em empresas de pequeno porte, não houve 
grandes problemas com a implantação deste programa de melhoria. Acredita-se que parte desse sucesso tenha ocorrido em função das pessoas da organização serem jovens (com idade média de 21 anos) e pouco experientes, e desta forma, aceitar paradigmas novos com maior facilidade do que quem atua no mercado de software há muitos anos.

Também acredita-se que a dificuldade de uso do modelo CMMI nas empresas de software possa ser atribuída a uma deficiência dos próprios cursos de graduação, que possuem poucas ou nenhuma disciplina específica da área de Qualidade de Software. Foi observado o não conhecimento do modelo em diversos contatos com outras empresas estabelecidas no mercado, inclusive clientes da empresa que foi realizado o presente estudo.

A empresa se mostrou autodidata em todas as fases da implantação do modelo, buscando sanar suas dúvidas através de listas de discussões ou contato com pessoas especializadas. Até o presente momento não foi contratada consultoria externa devido a restrições orçamentárias, porém sabe-se que esta consultoria seria de grande valor para a organização. $\mathrm{O}$ acesso fácil a informações da área pode ter facilitado todo o processo. Atualmente a empresa gerencia um portal da área de Qualidade de Software, onde diversas pessoas submetem seus trabalhos para serem armazenados. É possível que este aspecto tenha facilitado todo o trabalho e minimizado as resistências.

Atualmente a empresa não almeja ainda uma avaliação oficial devido às questões de custo, contudo, acredita-se hoje que o programa de melhoria de processo instituído é fator fundamental para a sobrevivência da mesma. A orientação da organização por processos e a satisfação do nível 2 de maturidade do CMMI são de extrema relevância para que os novos integrantes, em sua maioria alunos do segundo e terceiro período do curso de Ciência da Computação, possam ser melhor acompanhados e tenham seu trabalho inspecionado de tal forma a minimizar os problemas da construção de software.

\section{Bibliografia}

[CÂNDIDO 2004] Cândido, Edílson J. D. Uma simplificação da técnica análise de pontos de função para estimar tamanho de aplicativos web. Dissertação de Mestrado, USP, 2004.

[CMMI 2005] Capability Maturity Model Integration. Software Engineering Institute(SEI). Disponível em http://www.sei.cmu.edu/cmmi/.Acesso em 10/02/2005.

[HUMPHREY 1989] Humphrey W. S. Managing the software Process. AddisonWesley, 1989.

[IDEAL 2004] The IDEAL ${ }^{S M}$ Model. Software Engineering Institute(SEI). Disponível em http://www.sei.cmu.edu/ideal/ . Acesso em 10/12/2004.

[ISO 2004] The International Organization for Standardization and the International Electrotechnical Commission, ISO/IEC 15504 - Information Technology - Process Assessment - Part 1, 2004.

[MCT 2001] Ministério da Ciência e Tecnologia(MCT). Qualidade e produtividade no setor de software. Disponível em http://www.mct.gov.Br/Temas/info/Dsi/Quali2001/. Acesso em 01/12/2004.

[PMBOK 2000] Tradução livre do Project Management Body of Knowledge (PMBOK) 2000 realizada pelo PMI MG.

[PRESSMAN 2002] R. S. Pressman. Engenharia de Software, $5^{\mathrm{a}}$ ed. Rio de Janeiro, $M c$ Graw Hill, 2002.

[ROUILLER 2001] Rouiller, A. C. Gerenciamento de Projetos de Software para empresas de Pequeno Porte. Tese de Doutorado, UFPE, 2001.

[SOFTEX 2004] Sociedade para Promoção da Excelência do Software Brasileiro(Softex). Seção Qualidade de Software. Disponível em http://www.mct.gov.br/Temas/info/Dsi/qualidad/Qualidade.htm. Acesso em 05/12/2004. 